\title{
Understanding Interleaved Code
}

\author{
SPENCER RUGABER, KURT STIREWALT \\ College of Computing, Georgia Institute of Technology, Atlanta, GA \\ \{spencer,kurt\}@cc.gatech.edu \\ LINDA M. WILLS \\ linda.wills@ee.gatech.edu \\ School of Electrical and Computer Engineering, Georgia Institute of Technology, Atlanta, GA
}

\begin{abstract}
Complex programs often contain multiple, interwoven strands of computation, each responsible for accomplishing a distinct goal. The individual strands responsible for each goal are typically delocalized and overlap rather than being composed in a simple linear sequence. We refer to these code fragments as being interleaved. Interleaving may be intentional-for example, in optimizing a program, a programmer might use some intermediate result for several purposes-or it may creep into a program unintentionally, due to patches, quick fixes, or other hasty maintenance practices. To understand this phenomenon, we have looked at a variety of instances of interleaving in actual programs and have distilled characteristic features. This paper presents our characterization of interleaving and the implications it has for tools that detect certain classes of interleaving and extract the individual strands of computation. Our exploration of interleaving has been done in the context of a case study of a corpus of production mathematical software, written in Fortran from the Jet Propulsion Laboratory. This paper also describes our experiences in developing tools to detect specific classes of interleaving in this software, driven by the need to enhance a formal description of this software library's components. The description, in turn aids in the automated component-based synthesis of software using the library.
\end{abstract}

With every leaf a miracle.

- Walt Whitman.

Keywords: software understanding, interleaving, domain models, specification extraction, analysis tools.

\section{Introduction}

Imagine being handed a software system you have never seen before. Perhaps you need to track down a bug, rewrite the software in another language or extend it in some way. We know that software maintenance tasks such as these consume the majority of software costs (Boehm, 1981), and we know that reading and understanding the code requires more effort than actually making the changes (Fjeldstad and Hamlen, 1979). But we do not know what makes understanding the code itself so difficult.

Letovsky has observed that programmers engaged in software understanding activities typically ask "how" questions and "why" questions (Letovsky, 1988). The former require an in-depth knowledge of the programming language and the ways in which programmers express their software designs. This includes knowledge of common algorithms and data structures and even concerns style issues, such as indentation and use of comments. Nevertheless, the answers to "how" questions can be derived from the program text. "Why" questions are more troublesome. Answering them requires not only comprehending the program text but relating it to the program's purpose - solving some sort of problem. And 
the problem being solved may not be explicitly stated in the program text; nor is the rationale the programmer had for choosing the particular solution usually visible.

This paper is concerned with a specific difficulty that arises when trying to answer "why" questions about computer programs. In particular, it is concerned with the phenomenon of interleaving in which one section of a program accomplishes several purposes, and disentangling the code responsible for each purpose is difficult. Unraveling interleaved code involves discovering the purpose of each strand of computation, as well as understanding why the programmer decided to interleave the strands. To demonstrate this problem, we examine an example program in a step-by-step fashion, trying to answer the questions "why is this program the way it is?" and "what makes it difficult to understand?"

\subsection{NPEDLN}

The Fortran program, called NPEDLN, is part of the SPICELIB library obtained from the Jet Propulsion Laboratory and intended to help space scientists analyze data returned from space missions. The acronym nPEDLN stands for Nearest Point on Ellipsoid to Line. The ellipsoid is specified by the lengths of its three semi-axes (A, B, and C), which are oriented with the $x, y$, and $z$ coordinate axes. The line is specified by a point (LINEPT) and a direction vector (LINEDR). The nearest point is contained in a variable called PNEAR. The full program consists of 565 lines; an abridged version can be found in the Appendix with a brief description of subroutines it calls and variables it uses. The executable statements, with comments and declarations removed, are shown in Figure 1.

The lines of code in NPEDLN that actually compute the nearest point are somewhat hard to locate. One reason for this has to with error checking. It turns out that SP ICELIB includes an elaborate mechanism for reporting and recovering from errors, and roughly half of the code in NPEDLN is used for this purpose. We have indicated those lines by shading in Figure 2. The important point to note is that although it is natural to program in a way that intersperses error checks with computational code, it is not necessary to do so. In principal, an entirely separate routine could be constructed to make the checks and NPEDLN called only when all the checks are passed. Although this approach would require redundant computation and potentially more total lines of code, the resultant computations in NPEDLN would be shorter and easier to follow.

In some sense, the error handling code and the rest of the routine realize independent plans. We use the term plan to denote a description or representation of a computational structure that the designers have proposed as a way of achieving some purpose or goal in a program. This definition is distilled from definitions in (Letovsky and Soloway, 1986, Rich and Waters, 1990, Selfridge et al., 1993). Note that a plan is not necessarily stereotypical or used repeatedly; it may be novel or idiosyncratic. Following (Rich and Waters, 1990, Selfridge et al., 1993), we reserve the term cliché for a plan that represents a standard, stereotypical form, which can be detected by recognition techniques, such as (Hartman, 1991, Letovsky, 1988, Kozaczynski and Ning, 1994, Quilici, 1994, Rich and Wills, 1990, Wills, 1992). . Plans can occur at any level of abstraction from architectural overviews to code. By extracting the error checking plan from NPEDLN, we get the much smaller and, presumably, more understandable program shown in Figure 3. 


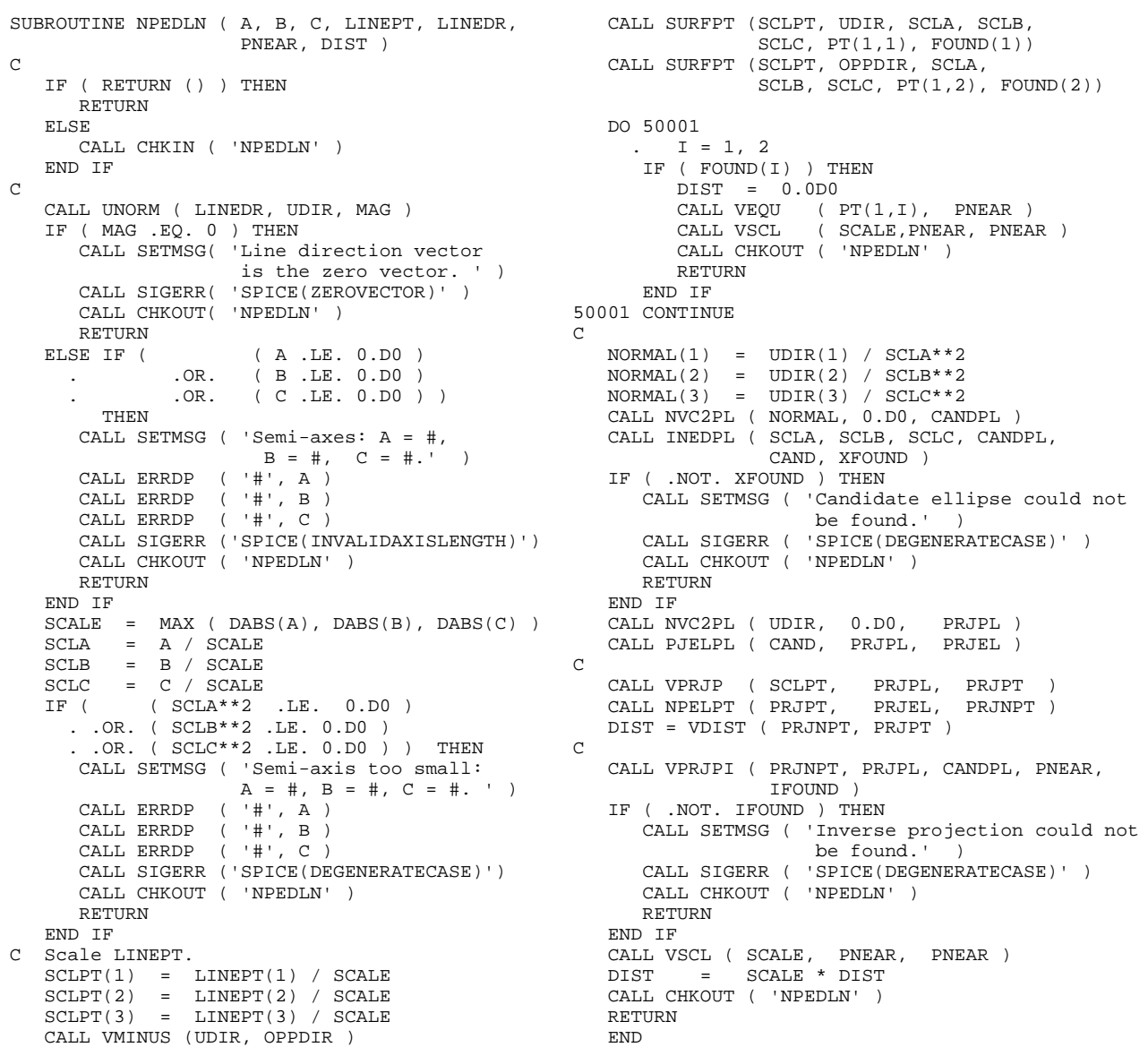

Figure 1. NPEDLN minus comments and declarations. 


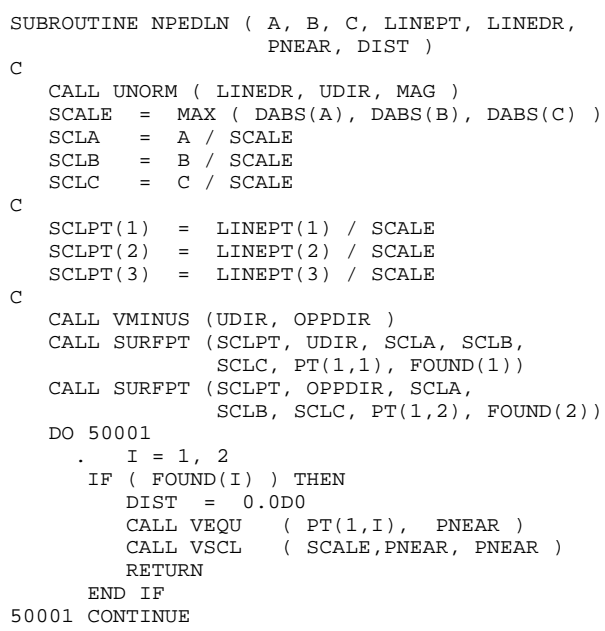

NORMAL $(1)=\operatorname{UDIR}(1) / \mathrm{SCLA} * \star 2$ NORMAL $(2)=\operatorname{UDIR}(2) / \mathrm{SCLB} * * 2$ NORMAL $(3)=\operatorname{UDIR}(3) / \mathrm{SCLC}^{\star *} 2$ CALL NVC2PL ( NORMAL, O.D0, CANDPL) CALL INEDPL ( SCLA, SCLB, SCLC, CANDPL, CAND, XFOUND)

Figure 3. The residual code without the error handling plan.

code responsible for doing this in NPEDLN is scattered throughout the program's text. It is highlighted in the excerpt shown in Figure 4.

The delocalized nature of this "scale-unscale" plan makes it difficult to gather together all the pieces involved for consistent maintenance. It also gets in the way of understanding the rest of the code, since it provides distractions that must be filtered out. Letovsky and Soloway's cognitive study (Letovsky and Soloway, 1986) shows the deleterious effects of delocalization on comprehension and maintenance.

When we extract the scale-unscale code from NPEDLN, we are left with the smaller code segment shown in Figure 5 that more directly expresses the program's purpose: computing the nearest point.

There is one further complication, however. It turns out that NPEDLN not only computes the nearest point from a line to an ellipsoid, it also computes the shortest distance between the line and the ellipsoid. This additional output (DIST) is convenient to construct because it can make use of intermediate results obtained while computing the primary output (PNEAR). This is illustrated in Figure 6. (The computation of DIST using VDIST is actually the last computation performed by the subroutine NPELPT, which NPEDLN calls; we have pulled this computation out of NPELPT for clarity of presentation.)

Note that an alternative way to structure SPICELIB would be to have separate routines for computing the nearest point and the distance. The two routines would each be more coherent, but the common intermediate computations would have to be repeated, both in the code and at runtime.

The "pure" nearest point computation is shown in Figure 7. It is now much easier to see the primary computational purpose of this code. 


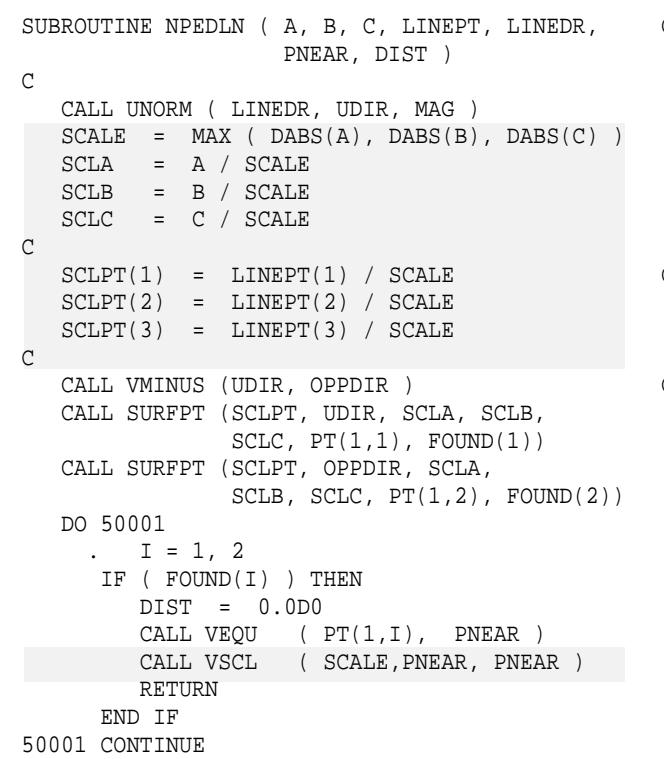

c NORMAL $(1)=\operatorname{UDIR}(1) / \operatorname{SCLA} * * 2$ NORMAL $(2)=\operatorname{UDIR}(2) / \mathrm{SCLB} * * 2$ NORMAL $(3)=\operatorname{UDIR}(3) / \mathrm{SCLC} * * 2$ CALL NVC2PL ( NORMAL, O.DO, CANDPL ) CALL INEDPL ( SCLA, SCLB, SCLC, CANDPL, CAND, XFOUND) CALL NVC2PL ( UDIR, O.DO, PRJPL ) CALL PJELPL ( CAND, PRJPL, PRJEL )

CALL VPRJP ( SCLPT, PRJPL, PRJPT ) CALL NPELPT ( PRJPT, PRJEL, PRJNPT) DIST $=$ VDIST $($ PRJNPT, PRJPT $)$

CALL VPRJPI ( PRJNPT, PRJPL, CANDPL, PNEAR, IFOUND )

CALL VSCL ( SCALE, PNEAR, PNEAR )

DIST $=$ SCALE * DIST

RETURN

END

Figure 4. Code with scale-unscale plan highlighted.

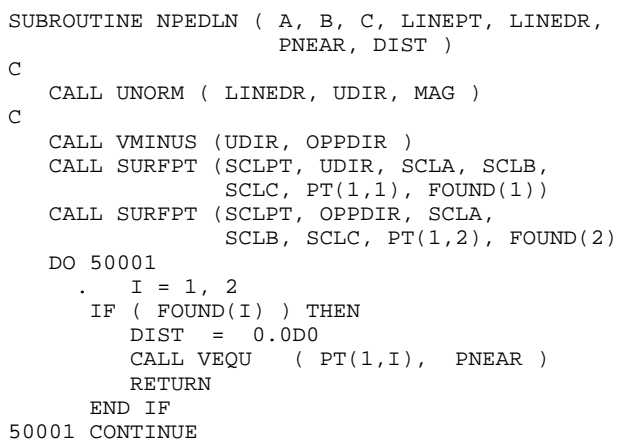

Figure 5. The residual code without the scale-unscale plan. 


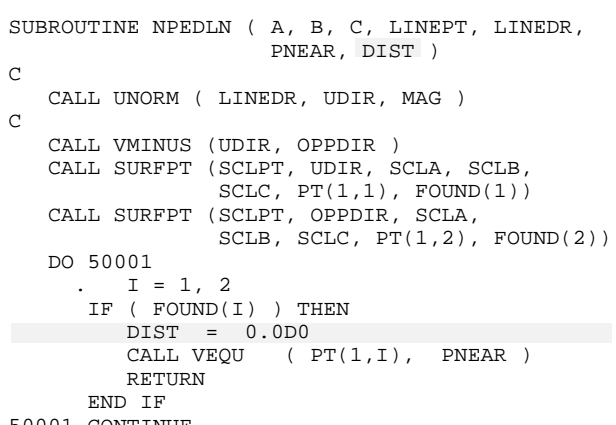

50001 CONTINUE

Figure 6. Code with distance plan highlighted.

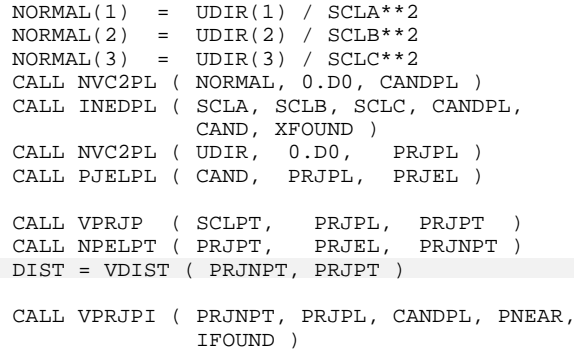

END

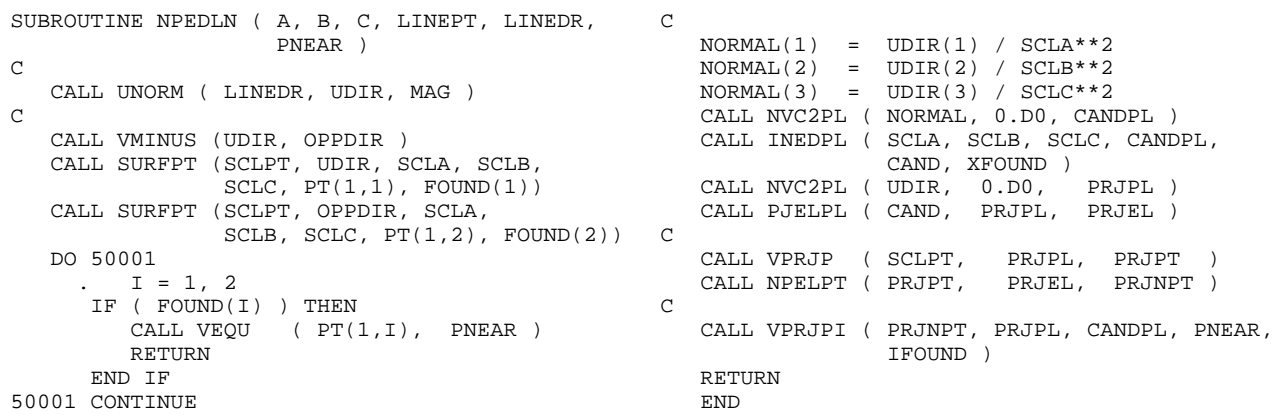

NORMAL $(1)=\operatorname{UDIR}(1) / \mathrm{SCLA} * * 2$

NORMAL $(2)=\operatorname{UDIR}(2) / \mathrm{SCLB} * * 2$

NORMAL (3) $=\operatorname{UDIR}(3), \mathrm{SCLC} * * 2$

NORMAL $=$ ULL NVC2PL ( NORMAL, 0.DO, CANDPL )

CALL NVC2PL ( NORMAL, O.DO, CANDPL)

CAND, XFOUND)

CALL NVC2PL ( UDIR, 0.D0, PRJPL ) C

Figure 7. The residual code without the distance plan. 
The production version of NPEDLN contains several interleaved plans. Intermediate Fortran computations are shared by the nearest point and distance plans. A delocalized scaling plan is used to improve numerical stability, and an independent error handling plan is used to deal with unacceptable input. Knowledge of the existence of the several plans, how they are related, and why they were interleaved is required for a deep understanding of NPEDLN.

\subsection{Contributions}

In this paper, we present a characterization of interleaving, incorporating three aspects that make interleaved code difficult to understand: independence, delocalization, and resource sharing. We have distilled this characterization from an empirical examination of existing software - primarily SPICELIB. Secondary sources of existing software which we also examined are a Cobol database report writing system from the US Army and a program for finding the roots of functions, presented and analyzed in (Basili and Mills, 1982) and (Rugaber et al., 1990). We relate our characterization of interleaving to existing concepts in the literature, such as delocalized plans (Letovsky and Soloway, 1986), coupling (Yourdon and Constantine, 1979), and redistribution of intermediate results (Hall, 1990, Hall, 1991).

We then describe the context in which we are exploring and applying these ideas. Our driving program comprehension problem is to elaborate and validate existing partial specifications of the JPL library routines to facilitate the automation of specification-driven generation of programs using these routines. We have developed analysis tools, based on the Software Refinery, to detect interleaving. We describe the analyses that we have formulated to detect specific classes of interleaving that are particularly useful in elaborating specifications. We then discuss open issues concerning requirements on software and plan representations that detection imposes, the role of application knowledge in addressing the interleaving problem, scaling up the scope of interleaving, and the feasibility of building tools to assist interleaving detection and extraction. We conclude with a description of how related research in cliché recognition as well as non-recognition techniques can play a role in addressing the interleaving problem.

\section{Interleaving}

Programmers solve problems by breaking them into pieces. Pieces are programming language implementations of plans, and it is common for multiple plans to occur in a single code segment. We use the term interleaving to denote this merging (Rugaber et al., 1995).

Interleaving expresses the merging of two or more distinct plans within some contiguous textual area of a program. Interleaving can be characterized by the delocalization of the code for the individual plans involved, the sharing of some resource, and the implementation of multiple, independent plans in the program's overall purpose. 
Interleaving may arise for several reasons. It may be intentionally introduced to improve program efficiency. For example, it may be more efficient to compute two related values in one place than to do so separately. Intentional interleaving may also be performed to deal with non-functional requirements, such as numerical stability, that impose global constraints which are satisfied by diffuse computational structures. Interleaving may also creep into a program unintentionally, as a result of inadequate software maintenance, such as adding a feature locally to an existing routine rather than undertaking a thorough redesign. Or interleaving may arise as a natural by-product of expressing separate but related plans in a linear, textual medium. For example, accessors and constructors for manipulating data structures are typically interleaved throughout programs written in traditional programming languages due to their procedural, rather than object-oriented structure. Interleaving cannot always be avoided (e.g., due to limitations of the available programming language) and may be desirable (e.g., for economy and avoiding duplication which can lead to inconsistent maintenance). Regardless of why interleaving is introduced, it complicates understanding a program. This makes it difficult to perform tasks such as extracting reusable components, localizing the effects of maintenance changes, and migrating to object-oriented languages.

There are several reasons interleaving is a source of difficulties. The first has to do with delocalization. Because two or more design purposes are implemented in a single segment of code, the individual code fragments responsible for each purpose are more spread out than they would be if they were segregated in their own code segments. Another reason interleaving presents a problem is that when it is the result of poorly thought out maintenance activities such as "patches" and "quick fixes", the original, highly coherent structure of the system may degrade. Finally, the rationale behind the decision to intentionally introduce interleaving is often not explicitly recorded in the program. For example, although interleaving is often introduced for purposes of optimization, expressing intricate optimizations in a clean and well-documented fashion is not typically done. For all of these reasons, our ability to comprehend code containing interleaved fragments is compromised.

Our goal is not to completely eliminate interleaving from programs, since that is not always desirable or possible to do at the level of source text. Rather, it is to find ways of detecting interleaving and representing the interleaved plans at a level of abstraction that makes the individual plans and their interrelationships clear.

We now examine each of the characteristics of interleaving - delocalization, sharing, independence - in more detail.

\subsection{Delocalization}

Delocalization is one of the key characteristics of interleaving: one or more parts of a plan are spatially separated from other parts by code from other plans with which they are interleaved. 


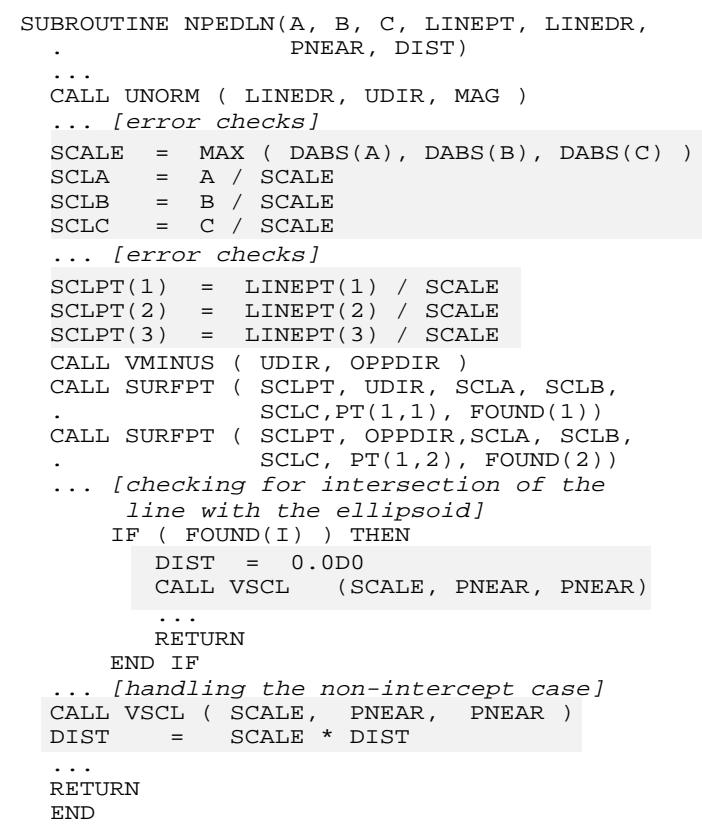

Figure 8. Portions of the NPEDLN Fortran program. Shaded regions highlight the lines of code responsible for scaling and unscaling. 
The "scale-unscale" pattern found in NPEDLN is a simple example of a more general delocalized plan that we refer to as a reformulation wrapper, which is frequently interleaved with computations in SPICELIB. Reformulation wrappers transform one problem into another that is simpler to solve and then transfer the solution back to the original situation. Other examples of reformulation wrappers in SPICELIB are reducing a three-dimensional geometry problem to a two-dimensional one and mapping an ellipsoid to the unit sphere to make it easier to solve intersection problems.

Delocalization may occur for a variety of reasons. One is that there may be an inherently non-local relationship between the components of the plan, as is the case with reformulation wrappers, which makes the spatial separation necessary. Another reason is that the intermediate results of part of a plan may be shared with another plan, causing the plans to overlap and their steps to be shuffled together; the steps of one plan separate those of the other. For example, in Figure 8, part of the unscale plan (computing the scaling factor) is separated from the rest of the plan (multiplying by the scaling factor) in all unscalings of the results (DIST and PNEAR). This allows the scaling factor to be computed once and the result reused in all scalings of the inputs $\mathrm{A}, \mathrm{B}$, and $\mathrm{C}$ and in unscaling the results.

Realizing that a reformulation wrapper or some other delocalized plan is interleaved with a particular computation can help prevent comprehension failures during maintenance (Letovsky and Soloway, 1986). It can also help detect when the delocalized plan is incomplete, as it was in an earlier version of our example subroutine whose modification history includes the following correction:

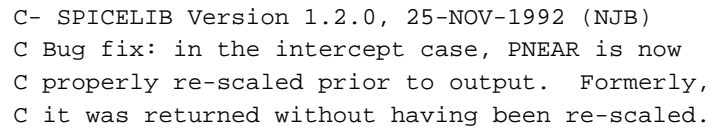

\subsection{Resource Sharing}

The sharing of some resource is characteristic of interleaving. When interleaving is introduced into a program, there is normally some implicit relationship between the interleaved plans, motivating the designer to choose to interleave them. An example of this within NPEDLN is shown in Figure 9. The shaded portions of the code shown are shared between the two computations for PNEAR and DIST. In this case, the common resources shared by the interleaved plans are intermediate data computations. The implementations for computing the nearest point and the shortest distance overlap in that a single structural element contributes to multiple goals.

The sharing of the results of some subcomputation in the implementation of two distinct higher level operations is termed redistribution of intermediate results by Hall (Hall, 1990, Hall, 1991). More specifically, redistribution is a class of function sharing optimizations which are implemented simply by tapping into the dataflow from some value producer and feeding it to an additional target consumer, introducing fanout into the dataflow. Redistribution covers a wide range of common types of function sharing optimizations, including common subexpression elimination and generalized loop fusion. Hall developed an automated technique for redistributing results for use in optimizing code generated from 


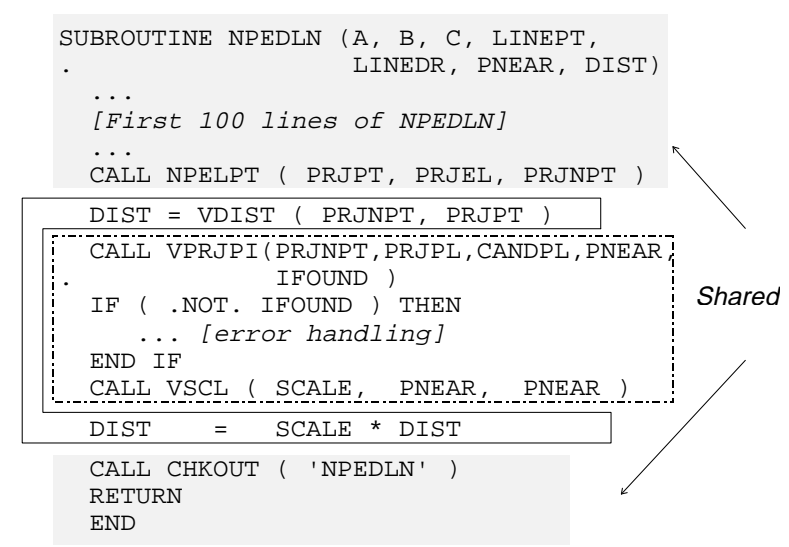

Figure 9. Portions of NPEDLN, highlighting two overlapping computations.

general-purpose reusable software components. Redistribution of results is a form of interleaving in which the resources shared are data values.

The commonality between interleaved plans might be in the form of other shared resources besides data values, for example control structures, lexical module structures, and names. Often when interleaving is unintentional, the resource shared is code space: the code statements of two plans are interleaved because they must be expressed in linear text. Typically, intentional interleaving involves sharing higher level resources.

Control coupling. Control conditions may be redistributed just as data values are. The use of control flags allows control conditions to be determined once but used to affect execution at more than one location in the program. In NPEDLN, for example, SURFPT is called to compute the intersection of the line with the ellipsoid. This routine returns a control flag, FOUND, indicating whether or not the intersection exists. This flag is then used outside of SURFPT to control whether the intercept or non-intercept case is to be handled, as is shown in Figure 10.

The use of control flags is a special form of control coupling: "any connection between two modules that communicates elements of control (Yourdon and Constantine, 1979)," typically in the form of function codes, flags, or switches (Myers, 1975). This sharing of control information between two modules increases the complexity of the code, complicating comprehension and maintenance.

Content coupling. Another form of resource sharing occurs when the lexical structure of a module is shared among several related functional components. For example, the entire contents of a module may be lexically included in another. This sometimes occurs when a programmer wants to take advantage of a powerful intraprocedural optimizer limited to improving the code in a single routine. Another example occurs when a programmer uses ENTRY statements to partially overlap the contents of several routines so that they may share 


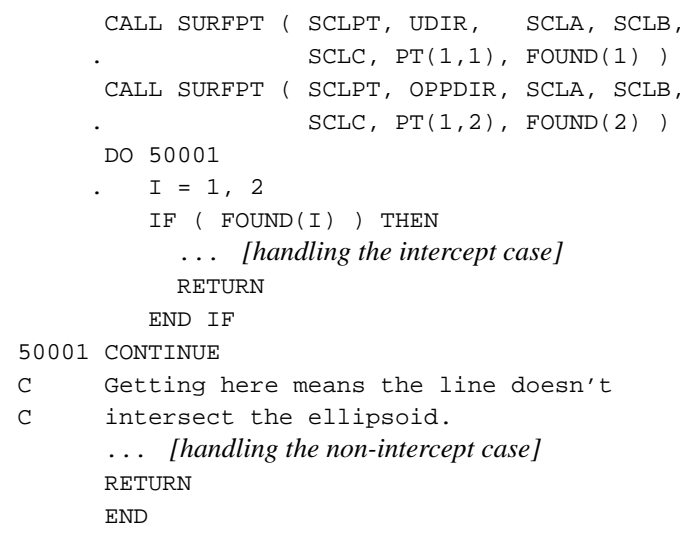

Figure 10. Fragment of subroutine showing control coupling

access to some state variables. This is sometimes done in a language, such as Fortran, that does not contain an encapsulation mechanism like packages or objects.

These two practices are examples of a phenomenon called content coupling (Yourdon and Constantine, 1979) in which - "some or all of the contents of one module are included in the contents of another" - and which often manifests itself in the form of a multipleentry module. Content coupling makes it difficult to independently modify or maintain the individual functions.

Name Sharing. A simple form of sharing is the use of the same variable name for two different purposes. This can lead to incorrect assumptions about the relationship between subcomputations within a program.

In general, the difficulty that resource sharing introduces is that it causes ambiguity in interpreting the purpose of program pieces. This can lead to incorrect assumptions about what effect changes will have, since the maintainer might be focusing on only one of the actual uses of the resource (variable, value, control flag, data structure slot, etc.).

\subsection{Independence}

While interleaving is introduced to take advantage of commonalities, it is also true that the interleaved plans each have a distinct purpose. Because understanding relates program goals to program code, having two goals realized in one section of code can be confusing.

There are several ways for dealing with this problem. One way would be to make two copies of the code segment, each responsible for one of the goals, and both duplicating any common code. In the NPEDLN example, a separate routine could be provided that is responsible for computing DIST. Although this may make understanding each of the routines somewhat simpler, there are costs due to the extra code and the implicit need, often forgotten, to update both versions of the common code whenever it needs to be fixed. A variant of 
this approach is to place the common code in a separate routine, replacing it in each of the two copies with a call to the new routine. This factoring approach works well when the common code is contiguous, but quickly becomes unworkable if the common code is interrupted by the plan specific code.

The bottom line is that this style of intentional interleaving confronts the programmer with a tradeoff between efficiency and maintainability/understandability. Ironically, making the efficiency choice may hinder efforts to make the code more efficient and reusable in the long run, such as parallelizing or "objectifying" the code (converting it to an object-oriented style).

\section{Case Study}

In order to better understand interleaving, we have undertaken a case study of production library software. The library, called SP ICELIB, consists of approximately 600 mathematical programs, written in Fortran by programmers at the Jet Propulsion Laboratory, for analyzing data sent back from space missions. The software performs calculations related to solar system geometry, such as coordinate frame conversions, intersections of rays, ellipses, planes, and ellipsoids, and light-time calculations. NPEDLN comes from this library.

We were introduced to SPICELIB by researchers at NASA Ames, who have developed a component-based software synthesis system called Amphion (Lowry et al., 1994, Lowry et al., 1994, Stickel et al., 1994). Amphion automatically constructs programs that compose routines drawn from SPICELIB. It does this by making use of a domain theory that includes formal specifications of the library routines, connecting them to the abstract concepts of solar system geometry. The domain theory is encoded in a structured representation, expressed as axioms in first-order logic with equality. A space scientist using Amphion can schematically specify the geometry of a problem through a graphical user interface, and Amphion automatically generates Fortran programs to call SP ICELIB routines to solve the described problem. Amphion is able to do this by proving a theorem about the solvability of the problem and, as a side effect, generating the appropriate calls. This is shown in the bottom half of Figure 11. Amphion has been installed at JPL and used by space scientists to successfully generate over one hundred programs to solve solar system kinematics problems. The programs consist of dozens of subroutine calls and are typically synthesized in under three minutes of CPU time using a Sun Sparc 2 (Lowry et al., 1994, Lowry et al., 1994).

Amphion's success depends on how accurate, consistent, and complete its domain theory is. An essential program understanding task is to validate the domain theory by checking it against the SPICELIB routines and extending it when incompletenesses are found. To do this, we need to be able to pull apart interleaved strands. For example, one incompleteness in Amphion's domain theory is that it does not fully cover the functionality of the routines in SPICELIB. Some routines compute more than one result. For example, NPEDLN computes the nearest point on an ellipsoid to a line as well as the shortest distance between that point and the ellipsoid. However, the domain theory does not describe both of these values. In the case of NPEDLN, only the nearest point computation is modelled, not the shortest distance. In these routines, it is often the case that the code responsible for the secondary functionalities is interleaved with the code for the primary function covered by 


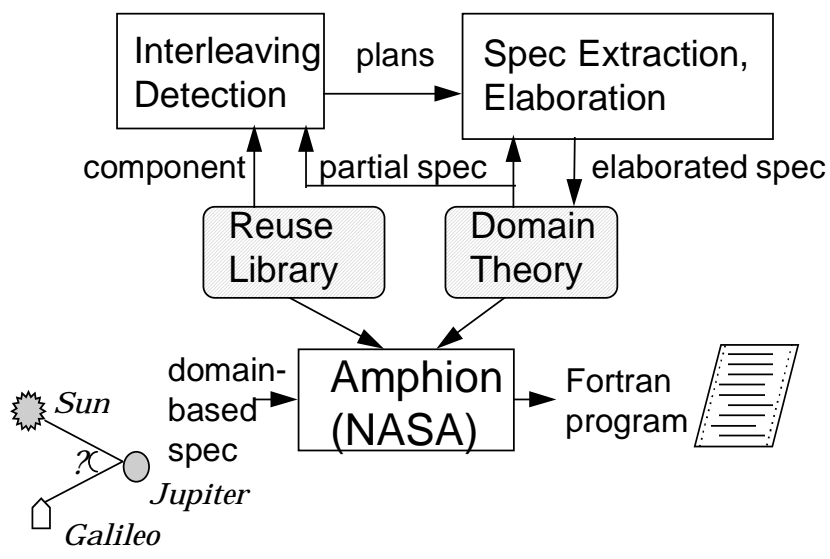

Figure 11. Applying interleaving detection to component-based reuse.

Amphion's domain theory. Uncovering the secondary functionality requires unraveling and understanding two interleaved computations.

Another way in which Amphion's current domain theory is incomplete is that it does not express preconditions on the use of the library routines; for example, that a line given as input to a routine must not be the zero vector or that an ellipsoid's semi-axes must be large enough to be scalable. It is difficult to detect the code responsible for checking these preconditions because it is usually tightly interleaved with the code for the primary computation in order to take advantage of intermediate results computed for the primary computation.

In collaboration with NASA Ames researchers, we explored ways in which Amphion's domain theory is incomplete, and we built program comprehension techniques to extend it. As the top half of Figure 11 shows, we developed mechanisms for detecting particular classes of interleaving, with the aim of extending the incomplete domain theory. In the process, we also performed analyses to gather empirical information about how much of SPICELIB is covered by the domain theory.

We have built interleaving detection mechanisms and empirical analyzers using a commercial tool called the Software Refinery (Reasoning Systems Inc.). This is a comprehensive tool suite including language-specific parsers and browsers for Fortran, C, Ada, and Cobol, language extension mechanisms for building analyzers for new languages, and a user interface construction tool for displaying the results of analyses. It maintains an object-oriented repository for holding the results of its analyses, such as abstract syntax trees and symbol tables. It provides a powerful wide-spectrum language, called Refine (Smith et al., 1985), which supports pattern matching and querying the repository. Using the Software Refinery allows us to leverage a commercially available tool as well as to evaluate the strengths and limitations of its approach to program analysis, which we discuss in Section 4.4. 


\subsection{Domain Theory Elaboration in Synthesis and Analysis}

Our motivations for validating and extending a partial domain theory of existing software come both from the synthesis and from the analysis perspectives. The primary motivations for doing this from the synthesis perspective are to make component retrieval more accurate in support of reuse, to assist in updating and growing the domain theory as new software components are added, and to improve the software synthesized.

From the software analysis perspective, the refinement and elaboration of the domain theory, based on what is discovered in the code, is a primary activity, driving the generation of hypotheses and informing future analyses. The process of understanding software involves two parallel knowledge acquisition activities (Brooks, 1983, Ornburn and Rugaber, 1992, Soloway and Ehrlich, 1984):

1. using domain knowledge to understand the code - knowledge about the application sets up expectations about how abstract concepts are typically manifested in concrete code implementations;

2. using knowledge of the code to understand the domain - what is discovered in the code is used to build up a description of various aspects of the application and to help answer questions about why certain code structures exist and what is their purpose with respect to the application.

We are studying interleaving in the context of performing these activities, given SPICELIB and an incomplete theory of its application domain. We are targeting our detection of interleaving toward elaborating the existing domain theory. We are also looking for ways in which the current knowledge in the domain theory can guide detection and ultimately comprehension.

\section{2. $\quad$ Extracting Preconditions}

Using the Software Refinery, we automated a number of program analyses, one of which is the detection of subroutine parameter precondition checks. A precondition is a Boolean guard controlling execution of a routine. Preconditions normally occur early in the code of a routine before a significant commitment (in terms of execution time and state changes that must be reversed) is made to execute the routine. Because precondition checks are often interspersed with the computation of intermediate results, they tend to delocalize the plans that perform the primary computational work. Moreover precondition computations are usually part of a larger plan that detects exceptional, possibly erroneous conditions in the state of a running program, and then takes alternative action when these conditions arise, such as returning with an error code, signaling, or invoking error handlers. In some instances the majority of the lines of code in a routine are there to deal with the preconditions and resulting exception handling rather than to actually implement the base plan of the routine.

We found many examples of precondition checks on input parameters in our empirical analysis of the SPICELIB. One such check occurs in the subroutine SURFPT and is shown in 


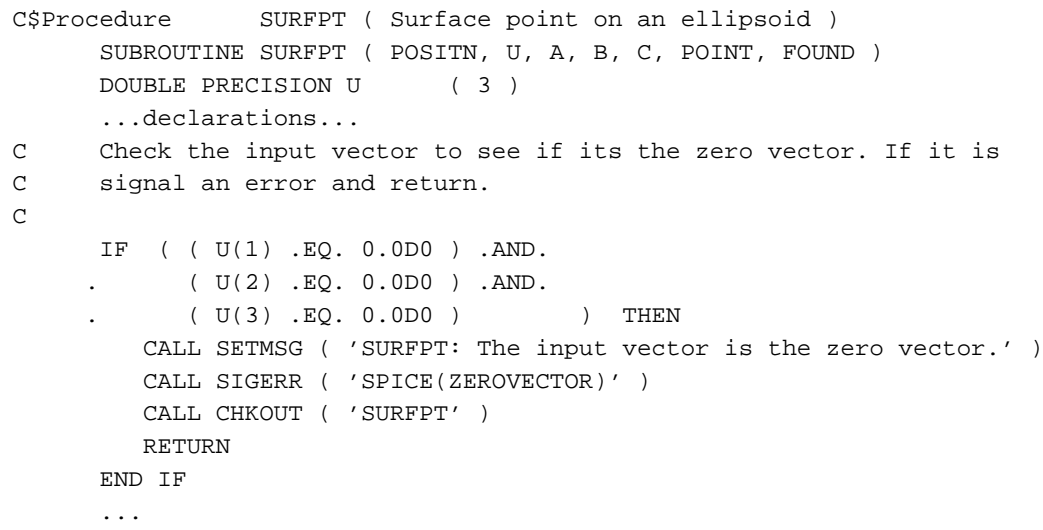

Figure 12. A fragment of the subroutine SURFPt in SPICELIB. This fragment shows a precondition check which invokes an exception if all of the elements of the $\mathrm{U}$ array are 0 .

Figure 12. SURFPT finds the intersection (POINT) of a ray (represented by a point POSITN and a direction vector $\mathrm{U}$ ) with an ellipsoid (represented as three semi-axes lengths $\mathrm{A}, \mathrm{B}$, and C), if such an intersection exists (indicated by FOUND). One of the preconditions checked by SURFPT is that the direction vector $\mathrm{U}$ is not the zero-vector.

Parameter precondition checks make explicit the assumptions a subroutine places on its inputs. The process of understanding a subroutine can be facilitated by detecting its precondition checks and using the information they encode to elaborate a high-level specification of the subroutine. We have created a tool that detects parameter precondition checks and extracts the preconditions into a documentation form suitable for expression as a partial specification. The specifications can then be compared against the Amphion domain model.

Precondition checks are particularly difficult to understand when they are sprinkled throughout the code of a subroutine as opposed to being concentrated at the beginning. However, we discovered that, though interleaved, these checks could be heuristically identified in SPICELIB by searching for IF statements whose predicates are unmodified input parameters (or simple dataflow dependents of them) and whose bodies invoke exception handlers. The logical negation of each of the predicates forms a conjunct in the precondition of the subroutine. The analysis that decides whether or not IF statements test only unmodified input parameters is specific to the Fortran language; but the analysis that decides if a code fragment is an exception plan depends on the fact that exceptions are dealt with in a stylized and stereotypical manner in SPICELIB. The implication is that the Fortran specific portion is not likely to need changing when we apply the tool to a new Fortran application; whereas the SPICELIB specific portion will certainly need to change. With this in mind, we chose a tool architecture that allows flexibility in keeping these different types of pattern knowledge separate and independently adaptable. 
Detecting Exception Handlers. In general, we need application specific knowledge about usage patterns in order to discover exception handlers. For example, the developers of SPICELIB followed a strict discipline of exception propagation by registering an exception upon detection using a subroutine SIGERR and then exiting the executing subroutine using a RETURN statement. Hence, a call to SIGERR together with a RETURN indicates a cliché for handling an exception in SPICELIB. In some other application, the form of this cliché will be different. It is, therefore, necessary to design the recognition component of our architecture around this need to specialize the tool with knowledge about the system being analyzed.

The Software Refinery provides excellent support for this design principle through the use of the rule construct and a tree-walker that applies these rules to an abstract syntax tree (AST). Rules declaratively specify state changes by listing the conditions before and after the change without specifying how the change is implemented. This is useful for including SPICELIB specific pattern knowledge because it allows the independent, declarative expression of the different facets of the pattern.

We recognize application specific exception handlers using two rules that search the AST for a call to SIGERR followed by a RETURN statement. These rules and the Refine code that applies them are presented in detail in (Rugaber et al., 1995).

Detecting Guards. Discovering guards, which are IF statements that depend only upon input parameters, involves keeping track of whether or not these parameters have been modified. If they have been modified before the check, then the check probably is not a precondition check on inputs. In Fortran, a variable $X$ can be modified by:

1. appearing on the left hand side of an assignment statement,

2. being passed into a subroutine which then modifies the formal parameter bound to $X$ by the call,

3. being implicitly passed into another subroutine in a common block and modified in this other subroutine, or

4. being explicitly aliased by an EQUIVALENCE statement to another variable which is then modified.

Currently our analysis does not detect modification through COMMON Or EQUIVALENCE because none of the code in SPICELIB uses these features with formal parameters. We track modifications to input parameters by using an approximate dataflow algorithm that propagates a set of unmodified variables through the sequence of statements in the subroutine. At each statement, if a variable $X$ in the set could be modified by the execution of the statement, then $X$ is removed from the set. After the propagation, we can easily check whether or not an IF statement is a guard.

Results. The result of this analysis is a table of preconditions associated with each subroutine. Since we are targeting partial specification elaboration for Amphion, we chose to make the tool output the preconditions in $\mathrm{LT}_{\mathrm{E}} \mathrm{X}$ form. Figure 13 gives examples of preconditions extracted for a few SPICELIB subroutines. Our tool generated the $\mathrm{LTT}_{\mathrm{E}} \mathrm{X}$ source included in Figure 13 without change. 


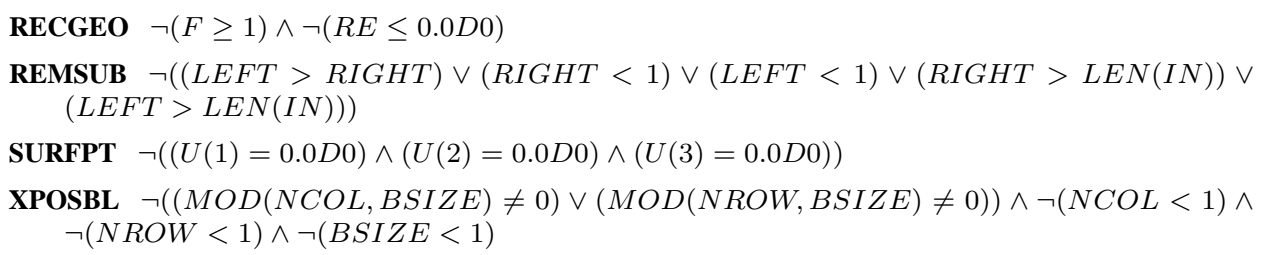

Figure 13. Preconditions extracted for some of the subroutines in SPICELIB.

Taken literally, the precondition for SURFPT, for example, states that one of the first three elements of the $\mathrm{U}$ array parameter must be non-zero. In terms of solar system geometry, $\mathrm{U}$ is seen as a vector, so the more abstract precondition can be stated as " $\mathrm{U}$ is not the zero vector." Extracting the precondition into the literal representation is the first step to being able to express the precondition in the more abstract form.

The other preconditions listed in Figure 13, stated in their abstract form, are the following. The subroutine RECGEO converts the rectangular coordinates of a point RECTAN to geodetic coordinates, with respect to a given reference spheroid whose equatorial radius is $\mathrm{RE}$, using a flattening coefficient $\mathrm{F}$. Its precondition is that the radius is greater than 0 and the flattening coefficient is less than 1 . The subroutine REMSUB removes the substring (LEFT:RIGHT) from a character string IN. It requires that the positions of the first character LEFT and the last character RIGHT to be removed are in the range 1 to the length of the string and that the position of the first character is less than the position of the last. Finally, the subroutine XPOSBL transposes the square blocks within a matrix вмAт. Its preconditions are that the block size BSIZE must evenly divide both the number of rows NROW in BMAT and the number of columns NCOL and that the block size, number of rows, and number of columns are all at least 1 .

\subsection{Finding Interleaving Candidates}

There are several other analyses that we have investigated using heuristic techniques for finding interleaving candidates. 


\subsubsection{Routines with Multiple Outputs}

One heuristic for finding instances of interleaving is to determine which subroutines compute more than one output. When this occurs, the subroutine is returning either the results of multiple distinct computations or a result whose type cannot be directly expressed in the Fortran type system (e.g., as a data aggregate). In the former case, the subroutine is realized as the interleaving of multiple distinct plans, as is the case with NPEDLN's computation of both the nearest point and the shortest distance.

In the latter case, the subroutine may be implementing only a single plan, but a maintainer's conceptual categorization of the subroutine is still obscured by the appearance of some number of seemingly distinct outputs. A good example of this case occurs in the SPICELIB subroutine SURFPT, which conceptually returns the intersection of a vector with the surface of an ellipsoid. However, it is possible to give SURFPT a vector and an ellipsoid that do not intersect. In such a situation the output parameter POINT will be undefined, but the Fortran type system cannot express the type: DOUBLE PRECISION $\vee$ Undefined. The original programmer was forced to simulate a variable of this type using two variables, POINT and FOUND, adopting the convention that when FOUND is false, the return value is Undefined, and when FOUND is true, the return value is POINT.

Clearly subroutines with multiple outputs complicate program understanding. We built a tool that determines the multiple output subroutines in a library by analyzing the direction of dataflow in parameters of functions and subroutines. A parameter's direction is either: in if the parameter is only read in the subroutine, out if the parameter is only written in the subroutine, or in-out if the parameter is both read and written in the subroutine. Multiple output subroutines will have more than one parameter with direction out or in-out.

Our tool bases its analysis on the structure chart (call graph) objects that the Software Refinery creates. The nodes of these structure charts are annotated with parameter direction information. The resulting analysis showed that 25 percent of the subroutines in SPICELIB had multiple output parameters. We were thus able to focus our work on these routines first, as they are likely to involve interleaving.

In addition, we performed an empirical analysis to determine, for those routines covered by the Amphion domain model (35 percent of the library), which ones have multiple output parameters, some of which are not covered by the domain model. We refer to outputs that are not mapped to anything in the domain model as dead end dataflows (similar to an interprocedural version of dead code (Aho et al., 1986)). Since the programs that Amphion creates can never make use of these return values, they have not been associated with any meaning in the domain theory. For example, NPEDLN's distance output (DIST) is a dead end dataflow as far as the domain theory is concerned. Dead end dataflows imply interleaving in the subroutine and/or an incompleteness in the domain theory. Our analysis revealed that of the subroutines covered by the domain theory, 30 percent have some output parameters that are dead end dataflows. These are good focal points for detecting interleaved plans that might be relevant to extending the domain theory. 


\subsubsection{Control Coupling}

Another heuristic for detecting potential interleaving finds candidate routines that may be involved in control coupling. Control coupling is often implemented by using a subroutine formal parameter as a control flag. So, we focus on calls to library routines that supply a constant as a parameter to other routines, as opposed to a variable. The constant parameter may be a flag that is being used to choose among a set of possible computations to perform. The heuristic strategy we use for detecting control coupling first computes a set of candidate routines that are invoked with a constant parameter at every call-site in the library or in code generated from the Amphion domain theory. Each member of this set is then analyzed to see if the formal parameter associated with the constant actual parameter is used to conditionally execute disjoint sections of code. Our analysis shows that 19 percent of the routines in SPICELIB are of this form.

\subsubsection{Reformulation Wrappers}

A third heuristic for locating interleaving is to ask: Which pairs of routines co-occur? Two routines co-occur if they are always called by the same routines, they are executed under the same conditions, and there is a flow of computed data from one to the other. We would like to detect co-occurrence pairs because they are likely to form reformulation wrappers. Of course, in general we would like to consider any code fragments as potential pairs, not just library routines. Once co-occurrence pairs are detected, they must be further checked to see whether they are inverses of each other. For example, in the "scale-unscale" reformulation wrapper, the operations that divide and multiply by the scaling factor co-occur and invert the effects of each other; the inputs are scaled (divided) and the results of the wrapped computation are later unscaled (multiplied). Through empirical investigation of SP ICELIB, we have discovered co-occurrence pairs that form reformulation wrappers and are building tools to perform this analysis automatically.

\section{Open Issues and Future Work}

We are convinced that interleaving seriously complicates understanding computer programs. But recognizing a problem is different from knowing how to fix it. Questions arise as to what form of representation is appropriate to hold the extracted information, how knowledge of the application domain can be used to detect plans, the extent to which the concept of interleaving scales up, and how powerful tools need to be to detect and extract interleaved components.

\subsection{Representation}

Our strategy for building program analysis tools is to formulate a program representation whose structural properties correspond to interesting program properties. A programming 
style tool, for example, uses a control flow graph that explicitly represents transfer of execution flow in programs. Irreducible control flow graphs signify the use of unstructured GO TO statements. The style tool uses this structural property to report violations of structured programming style. Since we want to build tools for interleaving detection we have to formulate a representation that captures the properties of interleaving. We do this by first listing structural properties that correspond to each of the three characteristics of interleaving and then searching for a representation that has these structural properties.

The key characteristics of interleaving are delocalization, resource sharing, and independence. In sequential languages like Fortran, delocalization often cannot be avoided when two or more plans share data. The components of the plans have to be serialized with respect to the dataflow constraints. This typically means that components of plans cluster around the computation of the data being shared as opposed to clustering around other components of the same plan. This total ordering is necessary due to the lack of support for concurrency in most high level programming languages. It follows then that in order to express a delocalized plan, a representation must impose a partial rather than a total execution ordering on the components of plans.

The partial execution ordering requirement suggests that some form of graphical representation is appropriate. Graph representations naturally express a partial execution ordering via implicit concurrency and explicit transfer of control and data. Since there are a number of such representations to choose from, we narrow the possibilities by noting that:

1. independent plans must be localized as much as possible, with no explicit ordering among them;

2. sharing must be detectable (shared resources should explicitly flow from one plan to another); similarly if two plans $p_{1}, p_{2}$ both share a resource provided by a plan $p_{3}$ then $p_{1}$ and $p_{2}$ should appear in the graph as siblings with a common ancestor $p_{3}$;

3. the representation must support multiple views of the program as the interaction of plans at various levels of abstraction, since interleaving may occur at any level of abstraction.

An existing formalism that meets these criteria is Rich's Plan Calculus (Rich, 1981, Rich, 1981, Rich and Waters, 1990). A plan in the Plan Calculus is encoded as a graphical depiction of the plan's structural parts and the constraints (e.g., data and control flow connections) between them. This diagrammatic notation is complemented with an axiomatized description of the plan that defines its formal semantics. This allows us to develop correctness preserving transformations to extract interleaved plans. The Plan Calculus also provides a mechanism, called overlays, for representing correspondences and relationships between pairs of plans (e.g., implementation and optimization relationships). This enables the viewing of plans at multiple levels of abstraction. Overlays also support a general notion of plan composition which takes into account resource sharing at all levels of abstraction by allowing overlapping points of view. 


\subsection{Exploiting Application Knowledge}

Most of the current technology available to help understand programs addresses implementation questions; that is, it is driven by the syntactic structure of programs written in some programming language. But the tasks that require the understanding - perfective, adaptive, and corrective maintenance - are driven by the problem the program is solving; that is, its application domain. For example, if a maintenance task requires extending NPEDLN to handle symmetric situations where more than one "nearest point" to a line exist, then the programmer needs to figure out what to do about the distance calculation also computed by NPEDLN. Why was DIST computed inside of the routine instead of separately? Was it only for efficiency reasons, or might the nearest point and the distance be considered a pair of results by its callers? In the former case, a single DIST return value is still appropriate, in the latter, a pair of identical values is indicated. To answer questions like these, programmers need to know which plans pieces of code are implementing. And this sort of plan knowledge derives from understanding the application area, not the program.

Another example from NPEDLN concerns reformulation wrappers. These plans are inherently delocalized. In fact, they only make sense as plans at all when considered in the context of the application: stable computations of solar system geometry. Without this understanding, the best hope is to recognize that the code has uniformly applied a function and its inverse in two places, without knowing why this was done and how the computations are connected.

The underlying issue is that any scheme for code understanding based solely on a top-down or a bottom-up approach is inherently limited. As illustrated by the examples, a bottom-up approach cannot hope to relate delocalized segments or disentangle interleavings without being able to relate to the application goals. And a top-down approach cannot hope to find where a plan is implemented without being able to understand how plan implementations are related syntactically and via dataflows. The implication is that a coordinated strategy is indicated, where plans generate expectations that guide program analysis and program analysis generates related segments that need explanation.

\subsection{Scaling the Concept of Interleaving}

We can characterize the ways interleaving manifests itself in source code along two spectrums. These form a possible design space of solutions to the interleaving problem and can help relate existing techniques that might be applicable. One spectrum is the scope of the interleaving, which can range from intraprocedural to interprocedural to object (clusters of procedures and data) to architectural. The other spectrum is the structural mechanism providing the interleaving, which may be naming, control, data, or protocol. Protocols are global constraints, such as maintaining stack discipline or synchronization mechanisms for cooperating processes. For example, the use of control flags is a control-based mechanism for interleaving with interprocedural scope. The common iteration construct involved in loop fusion is another control-based mechanism, but this interleaving has intraprocedural scope. Reformulation wrappers use a protocol mechanism, usually at the intraprocedural level, but they can have interprocedural scope. Multiple-inheritance is an example of a 
data-centered interleaving mechanism with object scope. Interleaving at the scope of objects and architectures or involving global protocol mechanisms is not yet well understood. Consequently, few mechanisms for detection and extraction currently exist in these areas.

\subsection{Tool Support}

We used the Software Refinery from Reasoning Systems in our analyses. This comprehensive toolkit provides a set of language-specific browsers and analyzers, a parser generator, a user interface builder, and an object-oriented repository for holding the results of analyses. We made particular use of two other features of the toolkit. The first is called the Workbench, and it provided pre-existing analyses for traditional graphs and reports such as structure charts, dataflow diagrams, and cross reference lists. The results of the analyses can be accessed from the repository using small, Refine language programs such as those described in (Rugaber et al., 1995). The Refine compiler was the other feature we used, compiling a Refine program into compiled Lisp.

The approach taken by the Refine language and tool suite has many advantages for attacking problems like ours. The language itself combines features of imperative, objectoriented, functional, and rule-based programming, thus providing flexibility and generality. Of particular value to us is its rule-based constructs. Before-and-after condition patterns define the properties of constructs without indicating how to find them. We had merely to add a simple tree walking routine to apply the rules to the abstract syntax tree. In addition to the rule-based features, Refine provides abstract data structures, such as sets, maps, and sequences, which manage their own memory requirements, thereby reducing programmer work. The object-oriented repository further reduces programmer responsibility by providing persistence and memory management.

We also take full advantage of Reasoning Systems' existing Fortran language model and its structure chart analysis. These allowed us a running start on our analysis and provided a robust handling of Fortran constructs that are not typically available from non-commercial research tools.

We can see several ways in which the Refine approach can be extended. In particular, the availability of other analyses, such as control flow graphs for Fortran and general dataflow analysis, would prove useful. Robust dataflow analysis is particularly important to the precision of precondition extraction.

\section{Related Work}

Techniques for detecting interleaving and disentangling interleaved plans are likely to build on existing program comprehension and maintenance techniques. 


\subsection{The Role of Recognition}

When what is interleaved is familiar (i.e., stereotypical, frequently used plans), cliché recognition (e.g., (Hartman, 1991, Johnson, 1986, Kozaczynski and Ning, 1994, Letovsky, 1988, Quilici, 1994, Rich and Wills, 1990, Wills, 1992) ) is a useful detection mechanism. In fact, most recognition systems deal explicitly with the recognition of clichés that are interleaved in specific ways with unrecognizable code or other clichés. One of the key features of GRASPR (Wills, 1992), for instance, is its ability to deal with delocalization and redistribution-type function sharing optimizations.

KBEmacs (Rich and Waters, 1990, Waters, 1979) uses a simple, special-purpose recognition strategy to segment loops within programs. This is based on detecting coarse patterns of data and control flow at the procedural level that are indicative of common ways of constructing, augmenting, and interleaving iterative computations. For example, KBEmacs looks for minimal sections of a loop body that have data flow feeding back only to themselves. This decomposition enables a powerful form of abstraction, called temporal abstraction, which views iterative computations as compositions of operations on sequences of values. The recognition and temporal abstraction of iteration clichés is similarly used in GRASPR to enable it to deal with generalized loop fusion forms of interleaving. Loop fusion is viewed as redistribution of sequences of values and treated as any other redistribution optimization (Wills, 1992).

Most existing cliché recognition systems tend to deal with interleaving involving data and control mechanisms. Domain-based clustering, as explored by DM-TAO in the DESIRE System (Biggerstaff et al., 1994), focuses on naming mechanisms, by keying in on the patterns of linguistic idioms used in the program, which suggest the manifestations of domain concepts.

Mechanisms for dealing with specific types of interleaving have been explicitly built into existing recognition systems. In the future, we envision recognition architectures that detect not only familiar computational patterns, but also recognize familiar types of transformations or design decisions that went into constructing the program. Many existing cliché recognition systems implicitly detect and undo certain types of interleaving design decisions. However, this process is usually done with special-purpose procedural mechanisms that are difficult to extend and that are viewed as having supporting roles to the cliché recognition process, rather than as being an orthogonal form of recognition.

\subsection{Disentangling Unfamiliar Plans}

When what is interleaved is unfamiliar (i.e., novel, idiosyncratic, not repeatedly used plans), other, non-recognition-based methods of delineation are needed. For example, slicing (Weiser, 1981, Ning et al., 1994) is a widely-used technique for localizing functional components by tracing through data dependencies within the procedural scope. Cluster analysis (Biggerstaff et al., 1994, Hutchens and Basili, 1985, Schwanke, 1991, Schwanke, 1989) is used to group related sections of code, based on the detection of shared uses of global data, control paths, and names. However, clustering techniques can only provide limited assistance by roughly delineating possible locations of functionally cohesive components. Another technique, called "potpourri module detection" (Calliss and Cornelius, 1990), de- 
tects modules that provide more than one independent service by looking for multiple proper subgraphs in an entity-to-entity interconnection graph. These graphs show dependencies among global entities within a single module. Presumably, the independent services reflect separate plans in the code.

Research into automating data encapsulation has recently provided mechanisms for hypothesizing possible locations of data plans at the object scope. For example, Bowdidge and Griswold (Bowdidge and Griswold, 1994) use an extended data flow graph representation, called a star diagram, to help programmers see all the uses of a particular data structure and to detect frequently occurring computations that are candidates for abstract functions. Techniques have also been developed within the $R E^{2}$ project (Canfora et al., 1993, Cimitile et al., 1994) for identifying candidate abstract data types and their associated modules, based on the call graph and dominance relations. Further research is required to develop techniques for extracting objects from pieces of data that have not already been aggregated in programmer-defined data structures. For example, detecting multiple pieces of data that are always used together might suggest candidates for data aggregation (as for example, in NPEDLN, where the input parameters A, B, and C are used as a tuple representing an ellipsoid, and the outputs PNEAR and DIST represent a pair of results related by interleaved, highly overlapping plans).

\section{Conclusion}

Interleaving is a commonly occurring phenomenon in the code that we have examined. Although a particular instance may be the result of an intentional decision on the part of a programmer trying to improve the efficiency of a program, it can nevertheless make understanding the program more difficult for subsequent maintainers. In our studies we have observed that interleaving typically involves the implementation of several independent plans in one code segment, often so that a program resource could be shared among the plans. The interleaving can, in turn, lead to each of the separate plan implementations being spread out or delocalized throughout the segment.

To investigate the phenomenon of interleaving, we have studied a substantial collection of production software, SPICELIB from the Jet Propulsion Laboratory. SPICELIB needs to be clearly understood in order to support automated program generation as part of the Amphion project, and we were able to add to the understanding by performing a variety of interleaving-based analyses. The results of these studies reinforce our feelings that interleaving is a useful concept when understanding is important, and that many instances of interleaving can be detected by relatively straightforward tools.

\section{Acknowledgments}

Support for this research has been provided by ARPA, (contract number NAG 2-890). We are grateful to JPL'S NAIF group for enabling our study of their SPICELIB software. We also benefited from insightful discussions with Michael Lowry at Nasa Ames Research Center concerning this study and interesting future directions. 


\section{Appendix NPELDN with Some of Its Documentation}

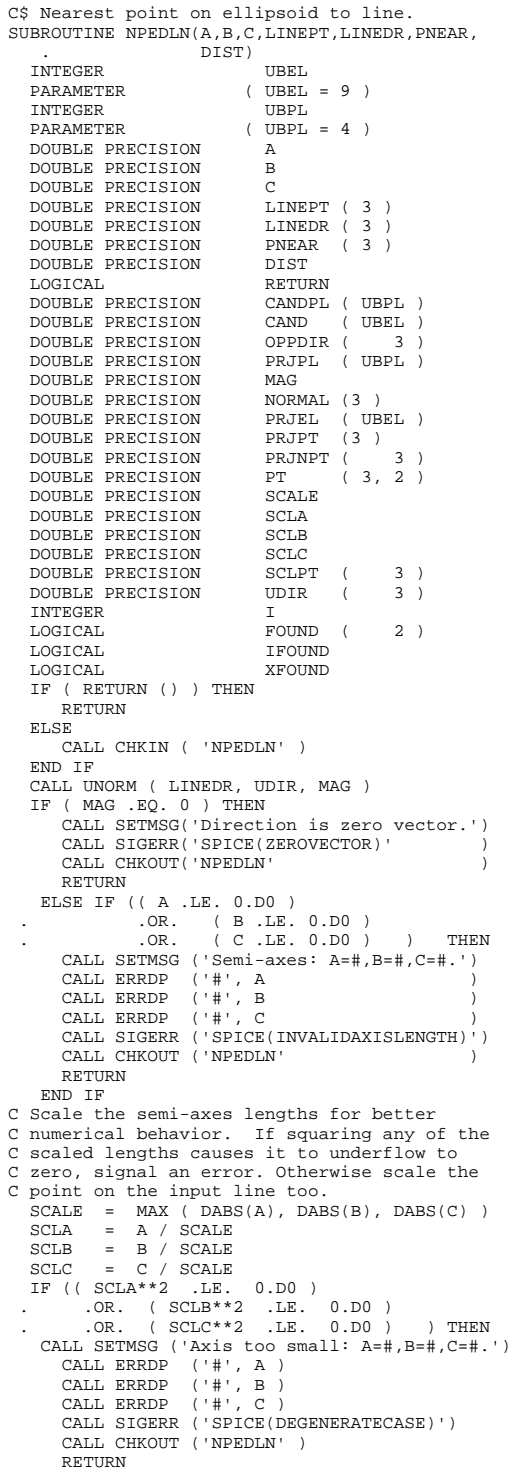




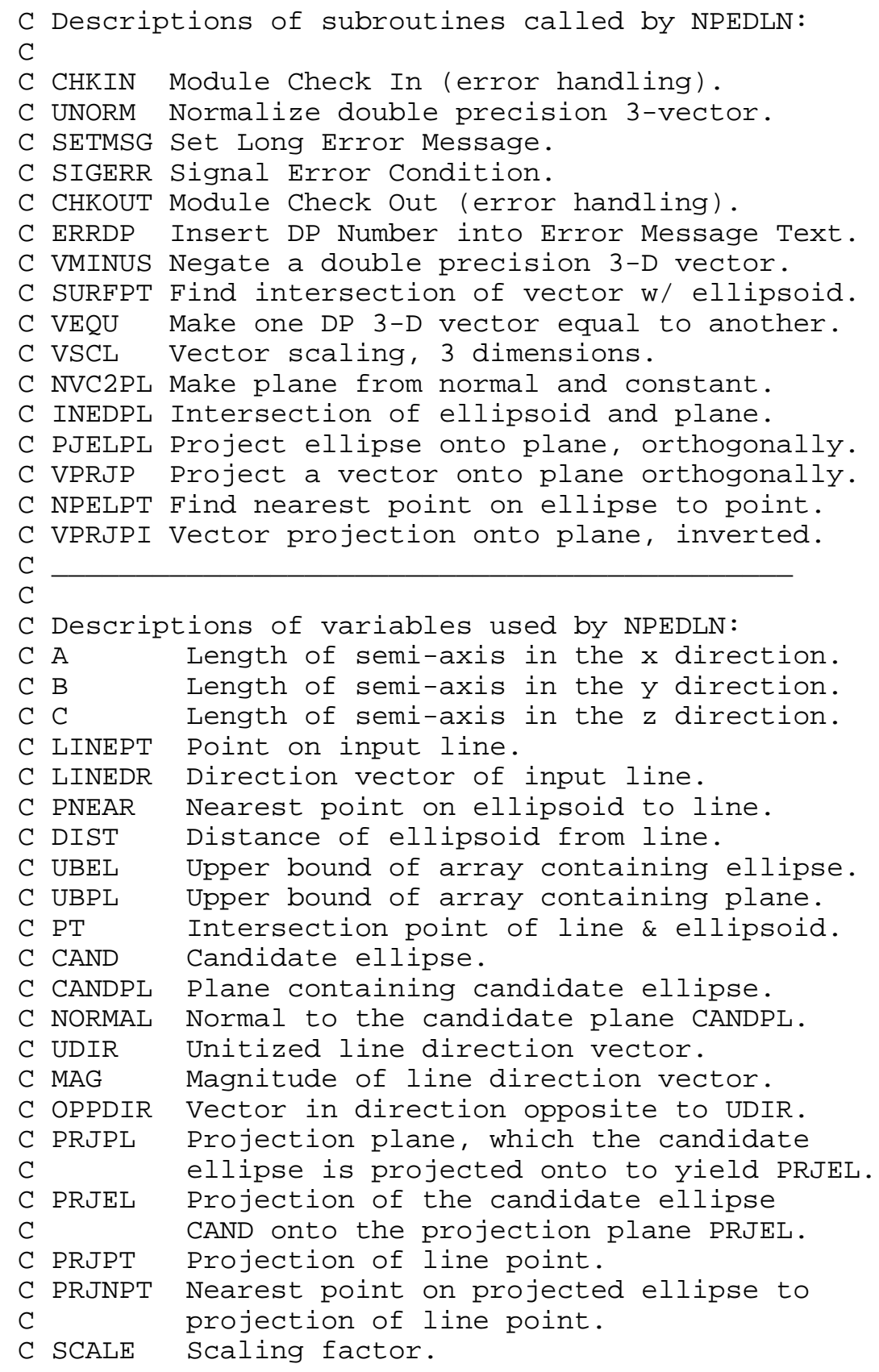




\section{References}

Aho, A., R. Sethi, and J. Ullman. Compilers: Principles, Techniques, and Tools. Addison-Wesley, Reading, MA, 1986.

Basili, V.R. and H.D. Mills. Understanding and documenting programs. IEEE Transactions on Software Engineering, 8(3):270-283, May 1982.

Biggerstaff, T., B. Mitbander, and D. Webster. Program understanding and the concept assignment problem. Communications of the ACM, 37(5):72-83, May 1994.

Boehm, Barry. Software Engineering Economics. Prentice Hall, 1981

Bowdidge, R. and W. Griswold. Automated support for encapsulating abstract data types. In Proc. 2nd ACM SIGSOFT Symposium on Foundations of Software Engineering, pages 97-110, New Orleans, Dec. 1994.

Brooks, R. Towards a theory of the comprehension of computer programs. International Journal of Man-Machine Studies, 18:543-554, 1983

Calliss, F. and B.Cornelius. Potpourri module detection. In IEEE Conf. on Software Maintenance - 1990, pages 46-51, San Diego, CA, November 1990. IEEE Computer Society Press.

Canfora, G., A.Cimitile, and M.Munro. A reverse engineering method for identifying reusable abstract data types. In Proc. of the First Working Conference on Reverse Engineering, pages 73-82, Baltimore, Maryland, May 1993. IEEE Computer Society Press.

Cimitile, A., M.Tortorella, and M.Munro. Program comprehension through the identification of abstract data types. In Proc. 3rd Workshop on Program Comprehension, pages 12-19, Washington, D.C., November 1994. IEEE Computer Society Press.

Fjeldstad, R.K. and W.T. Hamlen. Application program maintenance study: Report to our respondents. In GUIDE 48, 4 1979. Also appears in (Parikh and Zvegintozov, 1983).

Hall, R. Program improvement by automatic redistribution of intermediate results. Technical Report 1251, MIT Artificial Intelligence Lab., February 1990. PhD.

Hall, R. Program improvement by automatic redistribution of intermediate results: An overview. In M.Lowry and R.McCartney, editors, Automating Software Design. AAAI Press, Menlo Park, CA, 1991.

Hartman, J. Automatic control understanding for natural programs. Technical Report AI 91-161, University of Texas at Austin, 1991. PhD thesis.

Hutchens, D. and V.Basili. System structure analysis: Clustering with data bindings. IEEE Transactions on Software Engineering, 11(8), August 1985.

ReasoningSystems Incorporated. Software Refinery Toolkit. Palo Alto, CA.

Johnson, W.L. Intention-Based Diagnosis of Novice Programming Errors. Morgan Kaufmann Publishers, Inc., Los Altos, CA, 1986.

Kozaczynski, W. and J.Q. Ning. Automated program understanding by concept recognition. Automated Software Engineering, 1(1):61-78, March 1994.

Letovsky, S. Plan analysis of programs. Research Report 662, Yale University, December 1988. PhD.

Letovsky, S. and E.Soloway. Delocalized plans and program comprehension. IEEE Software, 3(3), 1986.

Lowry, M., A.Philpot, T.Pressburger, and I.Underwood. Amphion: automatic programming for subroutine libraries. In Proc. 9th Knowledge-Based Software Engineering Conference, pages 2-11, Monterey, CA, 1994.

Lowry, M., A.Philpot, T.Pressburger, and I.Underwood. A formal approach to domain-oriented software design environments. In Proc. 9th Knowledge-Based Software Engineering Conference, pages 48-57, Monterey, CA, 1994.

Myers, G. Reliable Software through Composite Design. Petrocelli Charter, 1975.

Ning, J.Q., A.Engberts, and W.Kozaczynski. Automated support for legacy code understanding. Communications of the ACM, 37(5):50-57, May 1994.

Ornburn, S. and S.Rugaber. Reverse engineering: Resolving conflicts between expected and actual software designs. In IEEE Conf. on Software Maintenance - 1992, pages 32-40, Orlando, Florida, November 1992.

Parikh, G. and N.Zvegintozov, editors. Tutorial on Software Maintenance. IEEE Computer Society, 1983. Order No. EM453.

Quilici, A. A memory-based approach to recognizing programming plans. Communications of the ACM, 37(5):84-93, May 1994.

Rich, C. A formal representation for plans in the Programmer's Apprentice. In Proc. 7th International Joint Conference on Artificial Intelligence, pages 1044-1052, Vancouver, British Columbia, Canada, August 1981. 
Rich, C. Inspection methods in programming. Technical Report 604, MIT Artificial Intelligence Lab., June 1981. $\mathrm{PhD}$ thesis.

Rich, C. and R.C. Waters. The Programmer's Apprentice. Addison-Wesley, Reading, MA and ACM Press, Baltimore, MD, 1990.

Rich, C. and L.M. Wills. Recognizing a program's design: A graph-parsing approach. IEEE Software, 7(1):82-89, January 1990.

Rugaber, S., S.Ornburn, and R.LeBlanc. Recognizing design decisions in programs. IEEE Software, 7(1):46-54, January 1990.

Rugaber, S., K.Stirewalt, and L.Wills. Detecting interleaving. In IEEE Conference on Software Maintenance 1995, pages 265-274, Nice, France, September 1995. IEEE Computer Society Press.

Rugaber, S., K.Stirewalt, and L.Wills. The interleaving problem in program understanding. In Proc. of the Second Working Conference on Reverse Engineering, pages 166-175, Toronto, Ontario, July 1995. IEEE Computer Society Press.

Schwanke, R. An intelligent tool for re-engineering software modularity. In IEEE Conference on Software Maintenance - 1991, pages 83-92, 1991.

Schwanke, R., R.Altucher, and M.Platoff. Discovering, visualizing, and controlling software structure. In Proc. 5th Int. Workshop on Software Specification and Design, pages 147-150, Pittsburgh, PA, 1989.

Selfridge, P., R.Waters, and E.Chikofsky. Challenges to the field of reverse engineering - A position paper. In Proc. of the First Working Conference on Reverse Engineering, pages 144-150, Baltimore, Maryland, May 1993. IEEE Computer Society Press.

Smith, D., G.Kotik, and S.Westfold. Research on knowledge-based software environments at Kestrel Institute. IEEE Transactions on Software Engineering, November 1985.

Soloway, E. and K.Ehrlich. Empirical studies of programming knowledge. IEEE Transactions on Software Engineering, 10(5):595-609, September 1984. Reprinted in C. Rich and R.C. Waters, editors, Readings in Artificial Intelligence and Software Engineering, Morgan Kaufmann, 1986.

Stickel, M., R.Waldinger, M.Lowry, T.Pressburger, I.Underwood, and A.Bundy. Deductive composition of astronomical software from subroutine libraries. In Proc. 12th International Conference on Automated Deduction, pages 341-55, Nancy, France, 1994.

Waters, R.C. A method for analyzing loop programs. IEEE Transactions on Software Engineering, 5(3):237-247, May 1979.

Weiser, Mark. Program slicing. In 5th International Conference on Software Engineering, pages 439-449, San Diego, CA, 31981.

Wills, L. Automated program recognition by graph parsing. Technical Report 1358, MIT Artificial Intelligence Lab., July 1992. PhD Thesis.

Yourdon, E. and L. Constantine. Structured Design: Fundamentals of a Discipline of Computer Program and Systems Design. Prentice-Hall, 1979 